\title{
A clinical implementation of in house produced acellular dermal matrix (ADM) inhabited by in vitro cultured cells - case series.
}

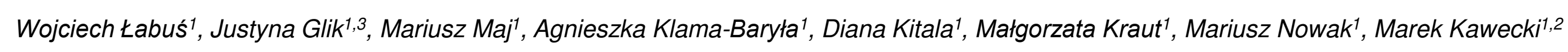

\author{
1 dr Stanislaw Sakiel Centre for Burn Treatment in Siemianowice Slaskie, Poland \\ 2 Department of Health Sciences, Technical-Humanistic Academy in Bielsko Biala, Poland \\ ${ }^{3}$ Unit for Chronic Wound Treatment Organization, Division of Nursery, School of Health Care, Medical University of Silesia, Poland
}

Background: An acellular dermal matrix (ADM) can be repopulated de novo by autologous or allogeneic skin cells. The goal was to evaluate a new burn treatment methods based on usage of self produced human living skin substitute.

\section{Material and methods:}

The proposed research programme has been approved by the Bioethics Committee of the Silesian Chamber of Physicians in Katowice.

For the production of acellular dermal matrices (ADM), allogeneic and biostatic human skin grafts deposited at the Tissue Bank of the Dr Stanisław Sakiel Centre for Burn Treatment (Polish: Centrum Leczenia Oparzeń, (CLO) were used. Allogeneic skin was harvested from deceased donors during multiorgan harvests, and then developed in accordance with applicable regulations.

Based on an analysis of the results obtained in the research work at the CLO, the optimal method for the production of stable, viable skin substitutes based on independently produced acellular dermal matrices $(\mathrm{ADM})$ and in vitro cultured skin cells.

The research protocol proposed an enzymatic method for cell removal $(0.05 \%$ trypsin solution in EDTAand $0.025 \%$ trypsin solution in EDTA).

Four patients were selected:

Case 1. A 63 year old patient with thermal burns of 34\% TBSA including 24\% III/IV. ADMs and allogeneic skin grafts (control) were meshed 1:2, grafted and then in vitro cultured autologous fibroblasts and keratinocytes suspended in PLRG were transplanted.

Case 2. A 36 year old patient with thermal burns of $24 \%$ TBSA including 24\% III/IV. ADMs and allogeneic skin grafts (control) were meshed 1:1,5, grafted and then in vitro cultured autologous fibroblasts and keratinocytes suspended in PLRG were transplanted on the donor sites wounds.

Case 3. A 63 year old patient with thermal burns of burns of $20 \%$ TBSA (IIa/IIb). ADMs were meshed 1:1,5, grafted and then in vitro cultured allogeneic fibroblasts and keratinocytes suspended in $0,9 \%$ $\mathrm{NaCl}$ were transplanted.

Case 4. A 59 year old patient with thermal burns of $31 \%$ TBSA (IIa/IIb). ADMs were meshed 1: 1.5 and then transplanted with in vitro cultured autologous fibroblasts and keratinocytes suspended in the PLRG.

\section{Results:}

Case 1: The patient received $2800 \mathrm{~cm}^{2}$ allogeneic skin grafts of which $1063 \mathrm{~cm}^{2}$ were ADMs. The number of grafted keratinocytes was 3.96 million with $90 \%$ vitality and 25.3 million fibroblasts with $96 \%$ vitality. In the day 0,3 and 5 the histochemical samples were prepared. In the day 6 patient died.

$$
\text { case } 1 \text { (day o) }
$$
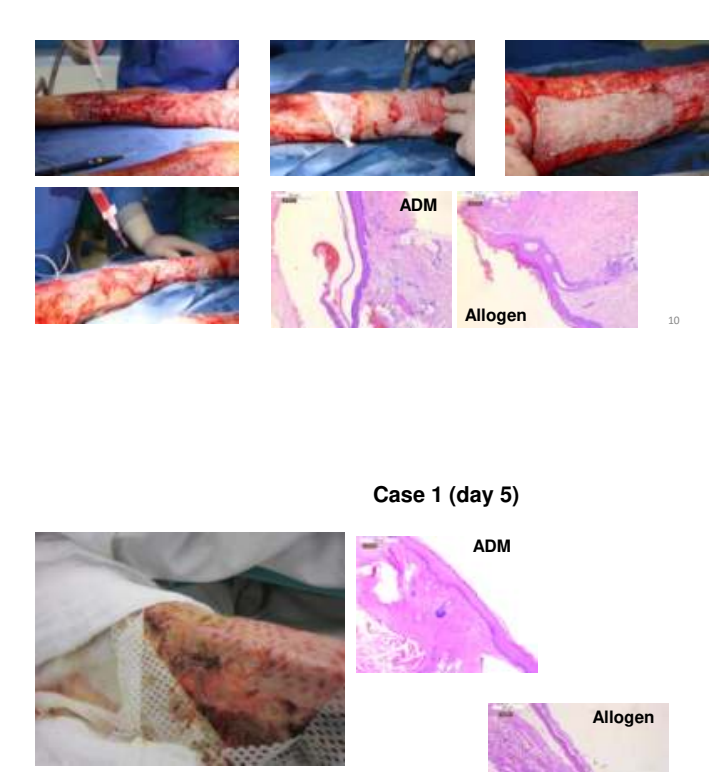

Case 1 (day 3)

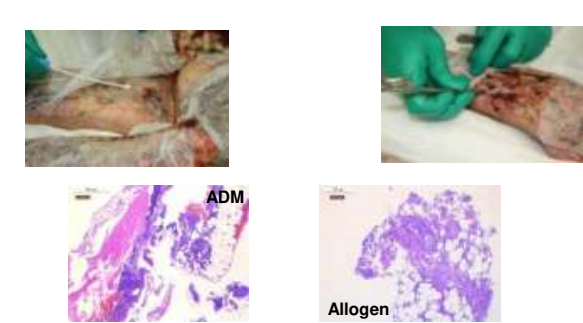

Case 1 (day 6, death)

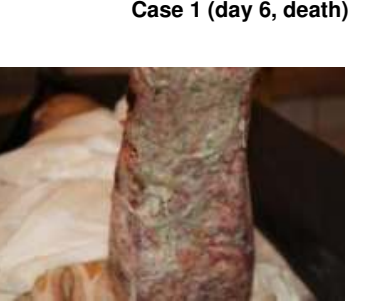

Case 2: The patient received $657 \mathrm{~cm}^{2}$ allogeneic skin grafts, meshed 1:1,5, of which $462 \mathrm{~cm}^{2}$ were ADMs. Keratinocytes and fibroblasts were suspended in PLRG. In the day 0, 3, 5, 14 and 21 the histochemical samples were done. Histochemical images depict the appropriate wound closure.

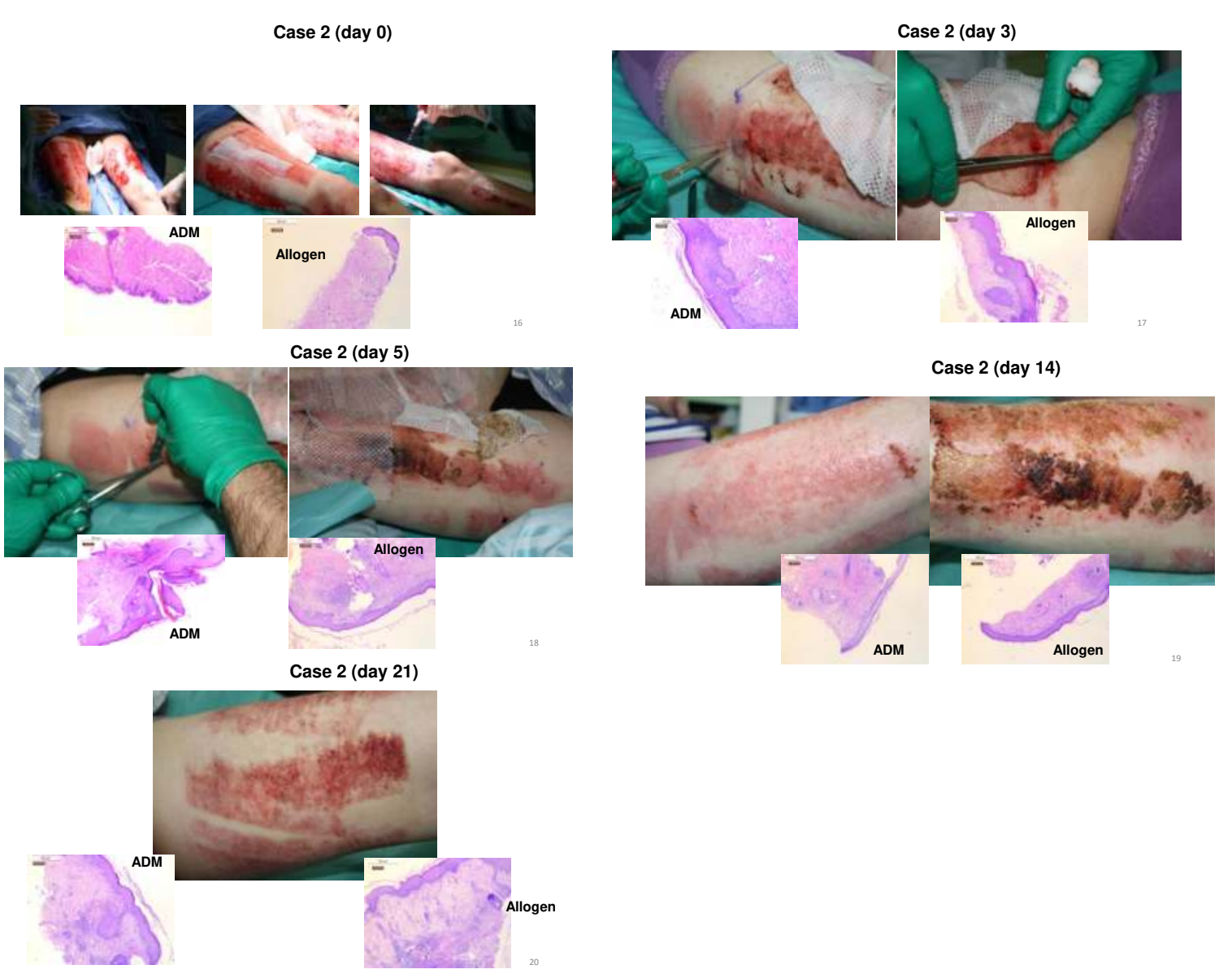

Case 3. The patient received $92 \mathrm{~cm} 2$ ADMs meshed 1:1,5. The allogeneic keratinocytes and fibroblasts were grafted in $0.9 \% \mathrm{NaCl}$. In the day 0 the histochemical sample was done. The patient did not consent to continue participation in the study.

Case 3

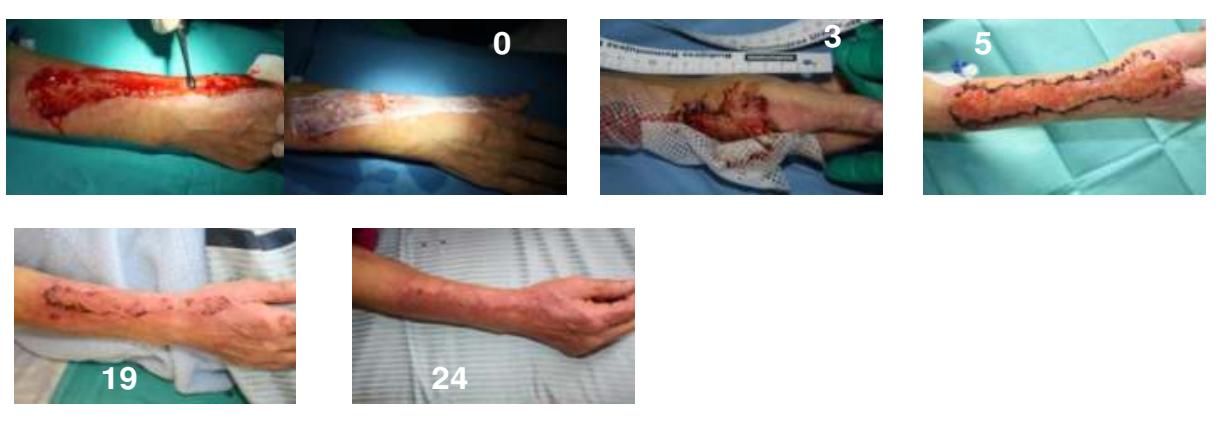

Case 4. The patient received $2,627 \mathrm{~cm}^{2}$ allogeneic skin grafts meshed 1:1.5 of which 2,525 $\mathrm{cm}^{2}$ were ADMs. ADM scaffolds were seeded with autologous keratinocytes and fibroblasts in PLRG. The patient had written off to the hospital.

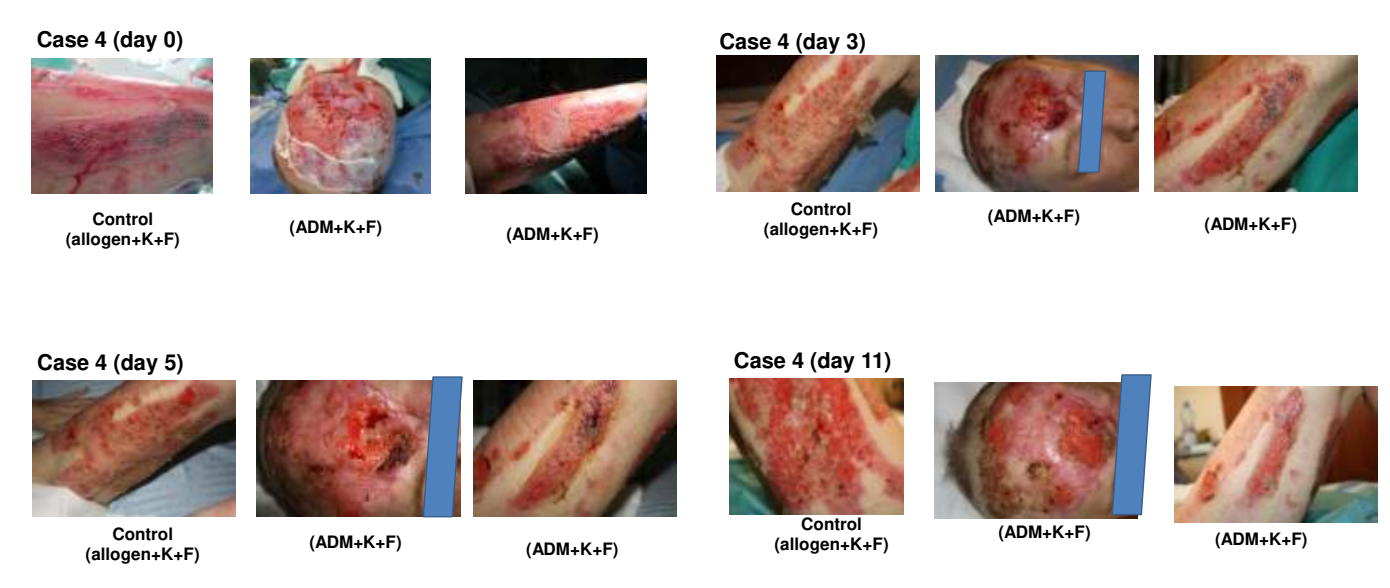

Conclusions:

The presented method for burn treatment may be the optimal method for burn wound therapy. However, it requires increased number of samples to perform statistical analysis of the results. 